\title{
Consequences of Confinement Conditions on Absorption in Molecular Nanofilms
}

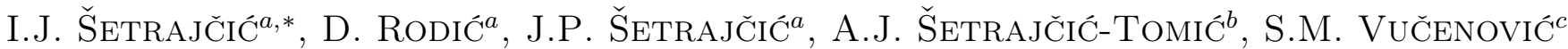 \\ ${ }^{a}$ University of Novi Sad, Faculty of Sciences, Department of Physics, 21000 Novi Sad, Vojvodina, Serbia \\ ${ }^{b}$ University of Novi Sad, Faculty of Medicine, Department of Pharmacy, 21000 Novi Sad, Vojvodina, Serbia \\ ${ }^{c}$ University of Banja Luka, Faculty of Sciences — Physics, 78000 Banja Luka, Republic of Srpska - B\&H
}

(Received December 11, 2015; in final form May 17, 2016)

This paper presents a model of molecular ultrathin crystalline film and analysis of dielectric properties of these spatially very restricted structures. Using the two-time dependent Green functions the energy spectrum and possible exciton states were determined and the dynamic permittivity was calculated. It was shown that the appearance of localized states in the boundary layers of the film depend on the thickness and the changing values of parameters in the border areas of the film. These localized states define schedule and determine the number of resonant absorption lines in the infrared area of the external electromagnetic radiation.

DOI: 10.12693/APhysPolA.133.129

PACS/topics: 71.35.-y; 71.35.Aa; 77.22.Ch; 77.55.-g; 78.20.Bh; 78.68. $+\mathrm{m} ; 81.07 . \mathrm{Nb}$

\section{Introduction}

Theoretical investigations of low-dimensional crystalline systems (nanostructures: ultrathin films, quantum wires, points, etc.) have been intensified recently in order to obtain fundamental information relating to extremely different physical and chemical properties of material, and also due to their wide practical application (technical and technological) in nano, opto, and bioelectronics [1-3]. Specificity of these "tiny" structures is that presence of near border planes causes highly changed general properties of these materials and occurrence of nonspecific phenomena (as a consequence of confinement effects [2]) in comparison to the properties of corresponding "large" samples [4-6].

This paper analyzes the influence of borderline filmstructure presence on energy spectrum of excitons (exciton dispersion law). Special attention was paid to the occurrence and spatial distribution of localized exciton states. In addition, optical properties were investigated (their dielectric permeability was determined). Results obtained were compared to the results of ideal infinite crystals, in order to find most important differences between these two systems.

Interest in the study of exciton subsystem appeared because excitons are responsible for dielectric, optic (absorption, dispersion, luminescence), photoelectric and other properties of crystals [7-9].

Analyses were done using the innovated method of Green functions, adapted for investigation of quantum structures [10-14]. Due to adequately incorporated statistics, this method is successfully applied in calculating both microscopic and macroscopic, balanced and imbalanced, properties of crystals.

*corresponding author; e-mail: igor.setrajcic@df.uns.ac.rs

\section{Exciton model}

Optic and dielectric properties of molecular crystal are dictated by the behavior of excitons of small concentration in the Frenkel exciton subsystem [7-9]. This model mirrors standard exciton Hamiltonian in configuration space

$$
\begin{aligned}
& H_{\mathrm{ex}}=H_{0}+\sum_{n} \Delta_{\boldsymbol{n}} P_{\boldsymbol{n}}^{+} P_{\boldsymbol{n}}+\sum_{\boldsymbol{n}, \boldsymbol{m}} X_{\boldsymbol{n} \boldsymbol{m}} P_{\boldsymbol{n}}^{+} P_{\boldsymbol{m}} \\
& \quad+\sum_{\boldsymbol{n}, \boldsymbol{m}} Y_{\boldsymbol{n} \boldsymbol{m}} P_{\boldsymbol{n}}^{+} P_{\boldsymbol{n}} P_{\boldsymbol{m}}^{+} P_{\boldsymbol{m}},
\end{aligned}
$$

where $P_{n}^{+}$and $P_{n}$ are creation and annihilation operators of excitons on node $\boldsymbol{n}$ in a crystal lattice. $\Delta_{n}$ is the energy of exciton localized at node $n$, while $X_{n, m}$ and $Y_{n, m}$ are matrix elements of exciton transfer from node $\boldsymbol{n}$ to node $\boldsymbol{m} \neq \boldsymbol{n}$.

Main difficulty in the exciton theory is the fact that Pauli operators $P^{+}$and $P$ are neither the Bose nor Fermi operators, but a certain hybrid of both [15], and their kinematics is fermionic for one node and bosonic for different nodes. For precise analysis of exciton systems, encompassing effects of inter-exciton interaction is not enough to simply replace the Pauli operators by the Bose operators. When studying dielectric response of the Frenkel excitons system with very small concentration on external perturbation (variable electromagnetic field) it is enough to use this rough approximation $[12,16]$ : $P, P^{+} \rightarrow B, B^{+}$. Thus, effective exciton Hamiltonian in harmonic approximation is ${ }^{\dagger}$

$$
H \equiv H_{\mathrm{eff}}=\sum_{n} \Delta_{n} B_{n}^{+} B_{n}+\sum_{n, \boldsymbol{m}} X_{\boldsymbol{n} m} B_{\boldsymbol{n}}^{+} B_{\boldsymbol{m}} .
$$

$\dagger$ It should be mentioned that in a huge amount of molecular crystals the optical and transfer properties depend on charge transfer (CT)/exciton (FE) coupling. For a model system of the Frenkel excitons in molecular crystals (of anthracene type) those couplings must be neglected [9]. 
Theoretical analysis will be performed using the commutation Green function [17-19]:

$$
\begin{gathered}
G_{\boldsymbol{n} \boldsymbol{m}}(t)=\left\langle\left\langle B_{\boldsymbol{n}}(t) \mid B_{\boldsymbol{m}}^{+}(0)\right\rangle\right\rangle= \\
\Theta(t)\left\langle\left[B_{\boldsymbol{n}}(t), B_{\boldsymbol{m}}^{+}(0)\right]\right\rangle,
\end{gathered}
$$

which satisfies equation of motion $[12,16,18]$ :

$$
\begin{aligned}
& \mathrm{i} \hbar \frac{\mathrm{d}}{\mathrm{d} t} G_{\boldsymbol{n} \boldsymbol{m}}(t)=\mathrm{i} \hbar \delta(t)\left\langle\left[B_{\boldsymbol{n}}(t), B_{\boldsymbol{m}}^{+}(0)\right]\right\rangle \\
& \quad+\Theta(t)\left\langle\left[B_{\boldsymbol{n}}(t), H\right] B_{\boldsymbol{m}}^{+}(0)-B_{\boldsymbol{m}}^{+}(0)\left[B_{\boldsymbol{n}}(t), H\right]\right\rangle .
\end{aligned}
$$

Using standard commutation relations for Bose operators [19], Eq. (2.4) now transforms to

$$
\begin{aligned}
& \mathrm{i} \hbar \frac{\mathrm{d}}{\mathrm{d} t} G_{\boldsymbol{n} \boldsymbol{m}}(t)=\mathrm{i} \hbar \delta(t) \delta_{\boldsymbol{n} \boldsymbol{m}}+\Delta_{\boldsymbol{n}} G_{\boldsymbol{n} \boldsymbol{m}}(t) \\
& \quad+\sum_{\boldsymbol{l}} X_{\boldsymbol{n} \boldsymbol{l}} G_{\boldsymbol{l m}}(t) .
\end{aligned}
$$

We solve this, by time differentiated, equation using time Fourier transformation

$$
\begin{gathered}
\delta(t)=\frac{1}{2 \pi} \int_{-\infty}^{+\infty} \mathrm{d} \omega \mathrm{e}^{-\mathrm{i} \omega t} ; \quad f_{\boldsymbol{a b}}(t)= \\
\int_{-\infty}^{+\infty} \mathrm{d} \omega f_{\boldsymbol{a} \boldsymbol{b}}(\omega) \mathrm{e}^{-\mathrm{i} \omega t},
\end{gathered}
$$

thus, we obtain

$$
\begin{aligned}
& \hbar \omega G_{\boldsymbol{n} \boldsymbol{m}}(\omega)=\frac{\mathrm{i} \hbar}{2 \pi} \delta_{\boldsymbol{n} \boldsymbol{m}}+\Delta_{\boldsymbol{n}} G_{\boldsymbol{n} \boldsymbol{m}}(\omega) \\
& +\sum_{\boldsymbol{l}} X_{\boldsymbol{n} \boldsymbol{l}} G_{\boldsymbol{l m}}(\omega) .
\end{aligned}
$$

For a crystal with primitive cubic lattice, we can use nearest neighbor approximation $\left(\boldsymbol{l} \rightarrow \boldsymbol{n} \pm \boldsymbol{\lambda}_{i}, i=x, y, z\right)$ : $\boldsymbol{n} \pm \boldsymbol{\lambda}_{1}=n_{x} \pm 1, n_{y}, n_{z} ; \boldsymbol{n} \pm \boldsymbol{\lambda}_{2}=n_{x}, n_{y} \pm 1, n_{z}$ and $\boldsymbol{n} \pm \boldsymbol{\lambda}_{3}=n_{x}, n_{y}, n_{z} \pm 1$, so equation above is transformed into

$$
\begin{aligned}
\hbar \omega & G_{n_{x} n_{y} n_{z}, m_{x} m_{y} m_{z}}(\omega)=\frac{\mathrm{i} \hbar}{2 \pi} \delta_{n_{x} n_{y} n_{z}, m_{x} m_{y} m_{z}} \\
& +\Delta_{n_{x} n_{y} n_{z}} G_{n_{x} n_{y} n_{z}, m_{x} m_{y} m_{z}}(\omega) \\
& +\left[X_{n_{x} n_{y} n_{z} ; n_{x}+1, n_{y} n_{z}} G_{n_{x}+1, n_{y} n_{z} ; m_{x} m_{y} m_{z}}(\omega)\right. \\
& +X_{n_{x} n_{y} n_{z} ; n_{x}-1, n_{y} n_{z}} G_{n_{x}-1, n_{y} n_{z} ; m_{x} m_{y} m_{z}}(\omega) \\
& +X_{n_{x} n_{y} n_{z} ; n_{x} n_{y}+1, n_{z}} G_{n_{x} n_{y}+1, n_{z} ; m_{x} m_{y} m_{z}}(\omega) \\
& +X_{n_{x} n_{y} n_{z} ; n_{x} n_{y}-1, n_{z}} G_{n_{x} n_{y}-1, n_{z} ; m_{x} m_{y} m_{z}}(\omega) \\
& +X_{n_{x} n_{y} n_{z} ; n_{x} n_{y} n_{z}+1} G_{n_{x} n_{y} n_{z}+1 ; m_{x} m_{y} m_{z}}(\omega) \\
& \left.+X_{n_{x} n_{y} n_{z} ; n_{x} n_{y} n_{z}-1} G_{n_{x} n_{y} n_{z}-1 ; m_{x} m_{y} m_{z}}(\omega)\right] .
\end{aligned}
$$

\section{Ultrathin exciton films}

Here we will observe very thin (several $\mathrm{nm}$ ) film with cubic crystalline structure, made on substrate by some technological process (steaming, reaching, spraying, etc.) [4-6]. The term ideal here is not used in the sense of unlimited space and presence of translational invariance for relevant energy parameters but in the sense that the inner structure is not violated by any means (no defects, impurities, etc.). Film dimensions are such that they are infinite in $X Y$ planes, while along $z$-axis has final thickness $(L)$. Therefore, this film has two infinite border areas parallel to $X Y$-plane, for: $z=0$ and $z=L \equiv N a$ (Fig. 1).

As a consequence of changed conditions on the borders of observed structure, we take that exciton energies in border $\left(n_{z}=0, n_{z}=N\right)$ and adjacent plane $\left(n_{z}=1\right.$, $\left.n_{z}=N-1\right)$ are perturbed, i.e.:

$$
\begin{aligned}
& \Delta_{\boldsymbol{n}} \equiv \Delta\left(1+d_{0} \delta_{n_{z}, 0}+d_{N} \delta_{n_{z}, N}\right), \\
& X_{\boldsymbol{n}, \boldsymbol{n}+\boldsymbol{\lambda}_{z}} \equiv X_{z}\left(1+x_{0} \delta_{n_{z}, 0}+x_{N} \delta_{n_{z}, N-1}\right), \\
& X_{\boldsymbol{n}, \boldsymbol{n}-\boldsymbol{\lambda} z} \equiv X_{z}\left(1+x_{0} \delta_{n_{z}, 1}+x_{N} \delta_{n_{z}, N}\right) .
\end{aligned}
$$

Values of parameter $d$ are defining energy change of isolated molecule at border area, and $x$ is energy perturbation in the exciton transfer from first inner atomic plane to border area, i.e. in border layers along $z$-axis.

Since border areas of the film are taken perpendicular to $z$-axis, index of parallel $X Y$ planes $n_{z}$ has values $n_{z}=0,1,2, \ldots N$, where $N \in[2,10]$ in ultra thin films. Indices $n_{x}$ and $n_{y}$, determining position of molecule in any $X Y$ plane, can have arbitrary whole-number values (practically from $-\infty$, to $+\infty$ ).

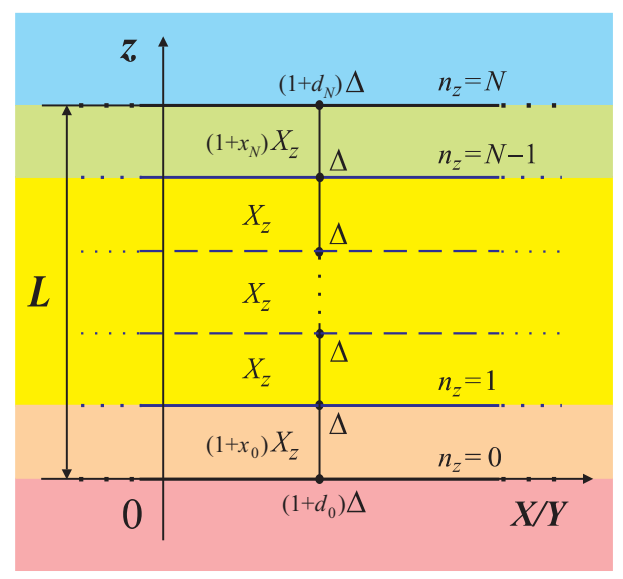

Fig. 1. Film cross-section in $X(Y) Z$ plane.

Due to a spatial limitation film along $z$-, to Eq. (2.8) only a partial spatial Fourier transformation may be applied (only along $x$ and $y$ axes):

$$
\begin{aligned}
& \delta_{\boldsymbol{n} \boldsymbol{m}}=\frac{1}{N_{x} N_{y}} \sum_{k_{x} k_{y}} \mathrm{e}^{\mathrm{i} k_{x} a_{x}\left(n_{x}-m_{x}\right)} \mathrm{e}^{\mathrm{i} k_{y} a_{y}\left(n_{y}-m_{y}\right)} \delta_{n_{z} m_{z}}, \\
& f_{\boldsymbol{n} \boldsymbol{m}}(\omega)=\frac{1}{N_{x} N_{y}} \sum_{k_{x} k_{y}} \mathrm{e}^{\mathrm{i} k_{x} a_{x}\left(n_{x}-m_{x}\right)} \mathrm{e}^{\mathrm{i} k_{y} a_{y}\left(n_{y}-m_{y}\right)} \\
& \quad \times f_{n_{z} m_{z}}\left(k_{x}, k_{y}, \omega\right) .
\end{aligned}
$$

In order to shorten notation, it is suitable to introduce next denotations $G_{n_{z} m_{z}} \equiv G_{n_{z} m_{z}}\left(k_{x}, k_{y}, \omega\right)$ and $\mathcal{K} \equiv \frac{\mathrm{i} \hbar}{2 \pi|X|}$. With $X_{x}=X_{y}=X_{z} \equiv-|X|$ and $a_{x}=a_{y}=a_{z} \equiv a$, we obtain 


$$
\begin{aligned}
& G_{n_{z}-1, m_{z}}\left(1+x_{0} \delta_{n_{z}, 1}+x_{N} \delta_{n_{z}, N}\right)+G_{n_{z}+1, m_{z}} \\
& \quad \times\left(1+x_{0} \delta_{n_{z}, 0}+x_{N} \delta_{n_{z}, N-1}\right)+G_{n_{z} m_{z}} \\
& \quad \times\left[\varrho-\frac{\Delta}{|X|}\left(d_{0} \delta_{n_{z}, 0}+d_{N} \delta_{n_{z}, N}\right)\right]=\mathcal{K} \delta_{n_{z}, m_{z}}
\end{aligned}
$$

by replacing

$$
\varrho=\frac{\hbar \omega-\Delta}{|X|}+2\left(\cos a k_{x}+\cos a k_{y}\right) .
$$

Equation (3.2) is in fact a system of $N+1$ nonhomogeneous algebraic-differential equations with (start-border) conditions: $G_{n_{z}, m_{z}}=0$, for $n_{z}<0$ and $n_{z}>N+1$.

\section{Dispersion law of excitons}

In order to find a spectrum of exciton energies, we need poles of the Green functions, which are obtained when a determinant of a system (3.2) is identically equalized to zero [12-14], i.e.

$$
\begin{aligned}
& D_{N+1}(\varrho)= \\
& \mid \begin{array}{cccccccc}
\varrho+\frac{\Delta}{|X|} d_{0} & 1+x_{0} & 0 & 0 & \cdots & 0 & 0 & 0 \\
1+x_{0} & \varrho & 1 & 0 & \cdots & 0 & 0 & 0 \\
0 & 1 & \varrho & 1 & \cdots & 0 & 0 & 0
\end{array} \\
& \begin{array}{c|c}
0 & \\
0 & \\
0 & \\
\cdot & \equiv 0 \\
0 & \\
1+x_{N} & \\
\varrho+\frac{\Delta}{|X|} d_{N} &
\end{array}
\end{aligned}
$$

From Eq. (3.3) the law of exciton dispersion: $E_{\boldsymbol{k}} \equiv$ $\hbar \omega(\boldsymbol{k})$ can be shown in non-dimensional form

$$
\begin{aligned}
& \mathcal{E}_{k_{x} k_{y}}(\nu)= \\
& \mathcal{F}_{x y}+\mathcal{G}_{z}(\nu),\left\{\begin{array}{l}
\mathcal{F}_{x y}=-2\left(\cos a k_{x}+\cos a k_{y}\right), \\
\mathcal{G}_{z}(\nu) \equiv \varrho_{\nu},
\end{array}\right.
\end{aligned}
$$

where $\varrho_{\nu}(\nu=1,2, \ldots N+1)$ are roots of upper determinant, depending on five parameters: number of atomic layers $N$ and in perturbation values $d_{0 / N}$ and $x_{0 / N}$. Calculation of roots, i.e. poles of the Green functions, due to impossibility of finding analytic solution (except in an ideal film case [12-14]), was found numerically and presented graphically (see Appendix A). The calculations are, due to the inability to find general analytical solutions, made with the help of adequate software (which we developed ourselves; see Appendix B for corresponding code lines).

Condition (4.1) will be solved for case of perturbed molecular film with five crystalline atomic planes along $z$ axis (two border planes and three within the film, i.e. 4 interatomic layers, therefore $N=4$ ). Border parameters will have changed values in the following intervals: $\left\{d_{0}, d_{N}\right\} \in[-0.3 ;+0.3]$ and $\left\{x_{0}, x_{N}\right\} \in[-0.8 ;+2.0]^{\ddagger}$. In this way we investigated and analyzed over 1200 different cases (with different values of parameters $d_{0}, d_{N}$, $x_{0}$ and $x_{N}$ and only one value of the parameter $N=4$ ).

In the numerical procedure we had to have control points. First, when the parameters were taking values $d_{0}=d_{N}=x_{0}=x_{N} \equiv 0$, we had to get analytically solvable case - ideal ultrathin film described in the previous footnote. We received other checkpoints from the symmetry of the problem, i.e. the model. Replacements: $d_{0} \rightleftarrows d_{N}$ and $x_{0} \rightleftarrows x_{N}$ had to have the same result because these operations would correspond to rotations or capsize of the film UP $\rightleftarrows$ DOWN. In all these situations, we were totally satisfied!

Important results of these calculations are shown in Figs. 2-4: ordinates show values for reduced nondimensional energies $\mathcal{E}_{k_{x} k_{y}}(\nu)$ depending on nondimensional function $\mathcal{F}_{x y}$ (on the abscissa) and parameter $\nu=1,2,3,4$ and 5 .

Figure 2 shows energy spectrum for excitons in fourlayered molecular film (full lines) when molecule energy changes at the node of border planes $\left(n_{z}=0\right.$ and $\left.n_{z}=N\right): \Delta_{0, N}=\left(1+d_{0, N}\right) \Delta$.
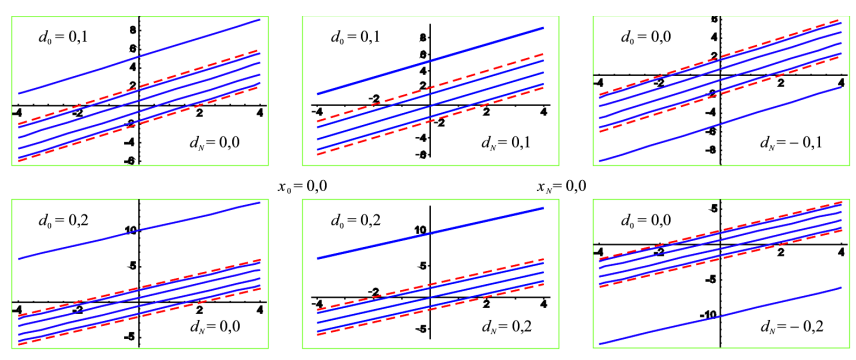

Fig. 2. Exciton energies of $d$-perturbed film.

Analyzing these graphs, we may conclude that by increasing (or decreasing) parameter $d$, spectrum of possible exciton energies widens, moving toward higher (or lower) energies. This moving occurs in such a way that one or two energy levels are "expelled" outside the bulk zone (marked by dotted lines). Levels are more distant from bulk zone if the perturbation is higher, i.e. if parameter $d$ is higher in the absolute value. These separated states are known as Tamm's or localized states [4-6].

\footnotetext{
$\ddagger$ Energies of isolated excitons may not be different for more than $\pm 30 \%$, since it would drastically disturb relation between $\Delta$ and $X$, and by that, we would deviate from basic presumptions of the Frenkel excitons. Transfer energies in border layers $(X)$ may not be reduced for more than $99.9 \dot{9} \%$, since in that case also there are no interactions of molecules from border planes and their adjacent, inner-film, planes. If we take $\left\{x_{0}, x_{N}\right\} \leq-1$, then the character of intermolecular interaction will change (attraction $\Leftrightarrow$ repulsion). If we take $\left\{x_{0}, x_{N}\right\} \geq 2$, that will strongly disrupt relation between $\Delta$ and $X$, and that will mean that a type of molecular crystal is changed where the Frenkel excitons are being formed [7-9] and those are the subject of consideration here.
} 
The number of localized states depends on the intensity of disturbance of the energy parameters of atoms from border areas.

Figure 3 shows reduced energies of excitons of fourlayered molecular film (full lines) when exciton transfer energy changes (intermolecular interaction) between border planes and adjacent planes $\left(n_{z}=0\right.$ and $n_{z}=1$, i.e. $n_{z}=N$ and $\left.n_{z}=N-1\right): X_{0, N}=\left(1+x_{0, N}\right) X$.

By analyzing these graphs it is possible to conclude that increase of parameter $x$ symmetrically widens spectrum of possible exciton energies, by the equal moving towards higher and lower energies. This widening occurs in the way that at the same time two or even four energy levels are "expelled" outside bulk zone (dotted lines). These levels of the localized states are further away from bulk zone if perturbation is higher, i.e. if $x$ parameter is higher. Lowering the parameter $x$ brings the minor narrowing inside energy levels, while width of whole spectrum stays practically the same and in this case there is no localized states.

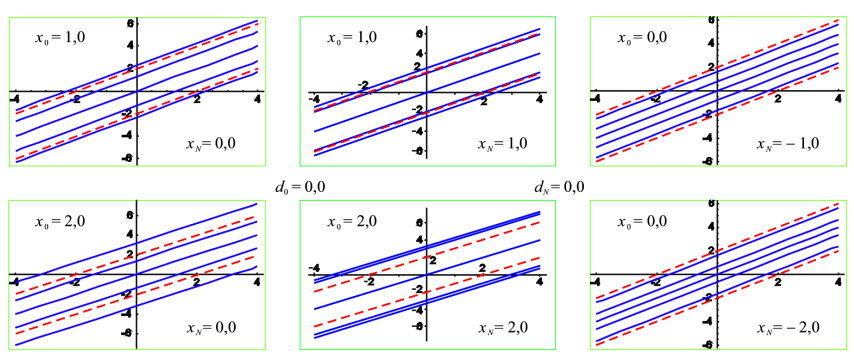

Fig. 3. Exciton energies of $x$-perturbed film.

Figure 4 shows reduced energies of excitons of fourlayered molecular film (full lines): left for totally unperturbed border parameters $\left(d_{0}=d_{N}=0 ; x_{0}=x_{N}=0\right)$ and right for the strongest perturbed border parameters $\left(d_{0}=d_{N}=0.3 ; x_{0}=x_{N}=2.0\right)$. In unperturbed case, there are no localized states [12], which are evident and strongly expressed for strong perturbation.
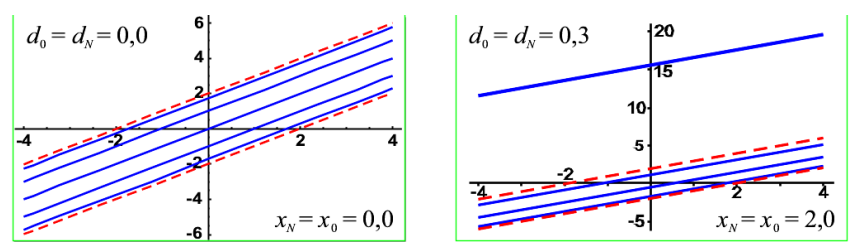

Fig. 4. Exciton energies of strongly perturbed film.

From all graphs, it is visible that the energy states of excitons in films are discrete. The opposite from continuous bulk zone, here can be as many energy levels as there are two-dimensional states, and we considered ultra thin film with 4 layers or 5 planes, i.e. with five possible energy levels. Localized states were found, but it is necessary to do a more detailed analysis of their spatial distribution and probability of their appearance. Also, when comparing Figs. 2 and 3 with Fig. 4, it is easy to find dominant influence of energy perturbation $\Delta$ (in comparison to $X$ ) and that effects caused by increase of parameter $x$ are silenced by presence of parameter $d$.

\section{Exciton states}

In order to determine the exciton Green functions, equation system (3.2) was represented in the matrix form

$$
\hat{\mathcal{D}}_{N+1} \tilde{\mathcal{G}}_{N+1}=\tilde{\mathcal{K}}_{N+1},
$$

where $\hat{\mathcal{D}}_{N+1}$ is the matrix corresponding to determinant of system $\mathcal{D}_{N+1}$, and $\tilde{\mathcal{G}}_{N+1}$ and $\tilde{\mathcal{K}}_{N+1}$ are vectors of the Green functions and Kronecker's deltas:

$$
\begin{gathered}
\tilde{\mathcal{G}}_{N+1}=\left(\begin{array}{c}
G_{0, m_{z}} \\
G_{1, m_{z}} \\
\vdots \\
G_{n_{z}, m_{z}} \\
\vdots \\
G_{N, m_{z}}
\end{array}\right) ; \\
\tilde{\mathcal{K}}_{N+1}=-\frac{\mathrm{i} \hbar}{2 \pi|X|}\left(\begin{array}{c}
\delta_{0, m_{z}} \\
\delta_{1, m_{z}} \\
\vdots \\
\delta_{n_{z}, m_{z}} \\
\vdots \\
\delta_{N, m_{z}}
\end{array}\right) .
\end{gathered}
$$

By acting with an inverse matrix $\hat{\mathcal{D}}_{N+1}^{-1}$, from the left side of Eq. (5.1), it follows:

$$
\tilde{\mathcal{G}}_{N+1}=\hat{\mathcal{D}}_{N+1}^{-1} \tilde{\mathcal{K}}_{N+1} \text {. }
$$

Since the inverse matrix can be expressed using adjunct one, where members $D_{i k}$ are co-factors of element $d_{i k}$ in direct matrix $D_{N+1}$, it can be written

$$
\begin{aligned}
G_{n_{z}, m_{z}}=\frac{1}{D_{N+1}} \sum_{q} D_{n_{z}, q} K_{q, m_{z}} & = \\
-\frac{1}{D_{N+1}} \frac{\mathrm{i} \hbar}{2 \pi|X|} \sum_{q} D_{n_{z}, q} \delta_{q, m_{z}} & =-\frac{\mathrm{i} \hbar}{2 \pi|X|} \frac{D_{n_{z}, m_{z}}}{D_{N+1}} .
\end{aligned}
$$

Considering that for balanced processes in a system only the diagonal Green functions are important $G_{n_{z} ; n_{z}}(\varrho) \equiv$ $G_{n_{z}}$, calculating co-factor $D_{n_{z}, m_{z}}(\varrho) \equiv D_{n_{z}}$ is significantly simplified

$$
D_{n_{z}}=B_{n_{z}}(\varrho) \times B_{N-n_{z}}(\varrho),
$$

$$
B_{n_{z}}=\left|\begin{array}{ccccccc}
\varrho+\frac{\Delta}{|X|} d_{0} & 1+x_{0} & 0 & \cdots & 0 & 0 & 0 \\
1+x_{o} & \varrho & 1 & \cdots & 0 & 0 & 0 \\
0 & 1 & \varrho & \cdots & 0 & 0 & 0 \\
. & . & . & \ddots & . & . & . \\
0 & 0 & 0 & \cdots & \varrho & 1 & 0 \\
0 & 0 & 0 & \cdots & 1 & \varrho & 1 \\
0 & 0 & 0 & \cdots & 0 & 1 & \varrho
\end{array}\right|_{n_{z}}
$$




$$
B_{N-n_{z}}=\left|\begin{array}{ccccccc}
\varrho & 1 & 0 & \cdots & 0 & 0 & 0 \\
1 & \varrho & 1 & \cdots & 0 & 0 & 0 \\
0 & 1 & \varrho & \cdots & 0 & 0 & 0 \\
\cdot & \cdot & . & \ddots & . & . & . \\
0 & 0 & 0 & \cdots & \varrho & 1 & 0 \\
0 & 0 & 0 & \cdots & 1 & \varrho & 1+x_{N} \\
0 & 0 & 0 & \cdots & 0 & 1+x_{N} & \varrho+\frac{\Delta}{|X|} d_{N}
\end{array}\right|_{N-n_{z}}
$$

with boundary conditions $B_{0}=B_{N-N}=1$.

The Green functions are multipolar [10-14], since the denominator is a polynomial $D_{N+1}$ of order $N+1$. Therefore, we must use the factorization of prime poles

$$
G_{n_{z}}=-\frac{\mathrm{i} \hbar}{2 \pi|X|} \sum_{\nu=1}^{N+1} \frac{g_{n_{z}}(\nu)}{\varrho-\varrho_{\nu}} .
$$

On the basis of (5.4) to (5.7), it follows that expression:

$$
g_{n_{z}}(\nu) \equiv g_{n_{z}}\left(\varrho_{\nu}\right)=\frac{B_{n_{z}}\left(\varrho_{\nu}\right) B_{N-n_{z}}\left(\varrho_{\nu}\right)}{\left.\frac{\mathrm{d}}{\mathrm{d} \varrho} D_{N+1}(\varrho)\right|_{\varrho=\varrho(\nu)}}
$$

represents spectral weights of the Green functions. These are in fact probabilities of presence of state with $\varrho_{\nu}$. Therefore, $g_{n_{z}}(\nu)$ enable determination of spatial distribution, i.e. probability of finding excitons with certain energies per layers of crystalline film. ${ }^{\S}$

Based on the formulae (5.5)-(5.8) it is clear that the statistical weights, and spatial distribution of probability for finding excitons states can be determined only numerically, because we have five parameters, with over 1200 different combinations of their values: $N=4$, $d_{0 / N} \in\{-0.3,+0.3\} \wedge x_{0 / N} \in\{-0.8,+2.0\}$ is done using the adequate software (see Appendix B) and graphically arranged.

In the following table with six sub-tables, values of reduced energies are represented with corresponding spectral functions (spatial distribution of probability) for four-layered film. In the case of the perturbed film, the spectral weights for given exciton energies can be found only by numerical methods, and results obtained for some values of perturbed parameters in the film are given for the center of Brillouin's two-dimensional zone $\left(k_{x}=k_{y}=0\right)$.

The first sub-table from the top gives spatial distribution for probabilities of presence of exciton energies

\footnotetext{
$\S$ This gives clear criterion for distinction between collective, bulk excitations (as described by flat and/or standing waves and have approximately the same probability of finding along whole section of a film) from localized excitations at the border areas (whose spectral weights show sharp maximum at the border perturbed layer and suddenly decrease within the film). Usual periodic dependence as a flat wave $\mathrm{e}^{\mathrm{i} k n}$ for voluminous states becoming declining exponential dependence $\mathrm{e}^{-\kappa n}$ for localized states, where basic condition is $\kappa \geq 0$. As said above, a condition in which that wave vector $\kappa$ takes imaginary values $\kappa=-\mathrm{i} k$, is reduced to the condition $|\varrho| \geq 2$, which shows that energies of localized states are outside continuous zone of bulk exciton energies.
}

in ideal exciton film (without perturbation of border parameters), see Appendix C. Other two sub-tables show spatial probabilities of finding exciton in the observed film when only $d_{0}$ or $d_{N}$ parameters are changing, while other perturbation parameters are equal to zero. The energies whose values are outside bulk zone (i.e. localized exciton states), as well as the highest values of probabilities (per film layers) for those energies, are shown in bold numbers. Increasing parameter $d_{0}$ shows that even spatial localization of excitons is increasing exactly at border areas of a film.

Next two sub-tables show spatial probabilities of finding excitons in the four-layered molecular film, but now for the case when only $x_{0}$ or $x_{N}$ parameters are changing, while other perturbation parameters are equal to zero. Both energies whose values are outside bulk zone (i.e. localized exciton states)and their highest values of probability (per layer of film) are also shown in bold numbers. For higher values of these parameters two or even four localized exciton states are present, with the highest spectral weights of excitons. Therefore, by increasing parameter $x$ probability of finding exciton at the border and first inner layers of ultra thin molecular film increases, so it is almost certain that exciton will be found at the first or second (or last and one before last) film layer. The last sub-table shows distribution of exciton states when border parameters are most perturbed. Presence of four localized states is evident with equal probabilities of finding exciton in the first border layer, i.e. at border areas and in the first inner planes. In addition, it is visible that parameter $d$ has dominant influence, since all effects obtained by parameter $x$ are silenced.

In order to overview these results, we have their graphic presentation — in Figs. 5 and 6 we will show the most interesting cases.

From all sub-tables (in Table I) and from all graphs (in Figs. 5 and 6 ) it is visible that at every node of a crystalline layer there is exactly one exciton, which may be in $N+1=5$ different energy states, with different probabilities of finding. For arbitrary number of atomic planes in film $(N+1)$ the following relation is valid, pointing that a sum of all probabilities for finding exciton is equal to the number of possible states

$$
\sum_{\nu=1}^{N+1} \sum_{n_{z}=0}^{N} g_{n_{z}}^{\nu}\left(\varrho_{\nu}\right)=N+1,
$$

which include the both bulk (voluminous) and localized states.

\section{Ultrathin films permittivity}

To determine the crystalline film dynamic permittivity, as in [10-14], we will use formula of DzyaloshinskiPitaevski [18]:

$$
\varepsilon_{n_{z}}^{-1}(\omega)=1-\frac{\pi S|X|}{\mathrm{i} \hbar}\left[G_{n_{z}}(\omega)+G_{n_{z}}(-\omega)\right] .
$$

with a note that permittivity here depends on the position of film plane $\left(n_{z}\right)$. Substituting expression for the 

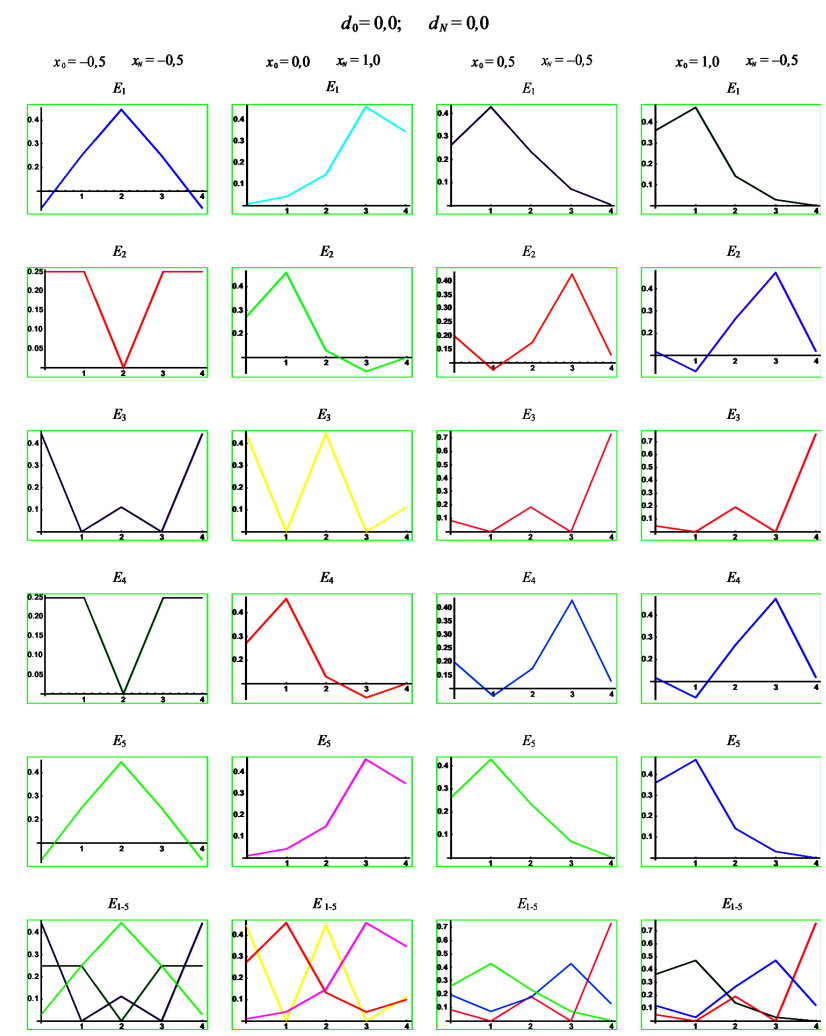

Fig. 5. Space distribution of exciton states in ultrathin molecular film.
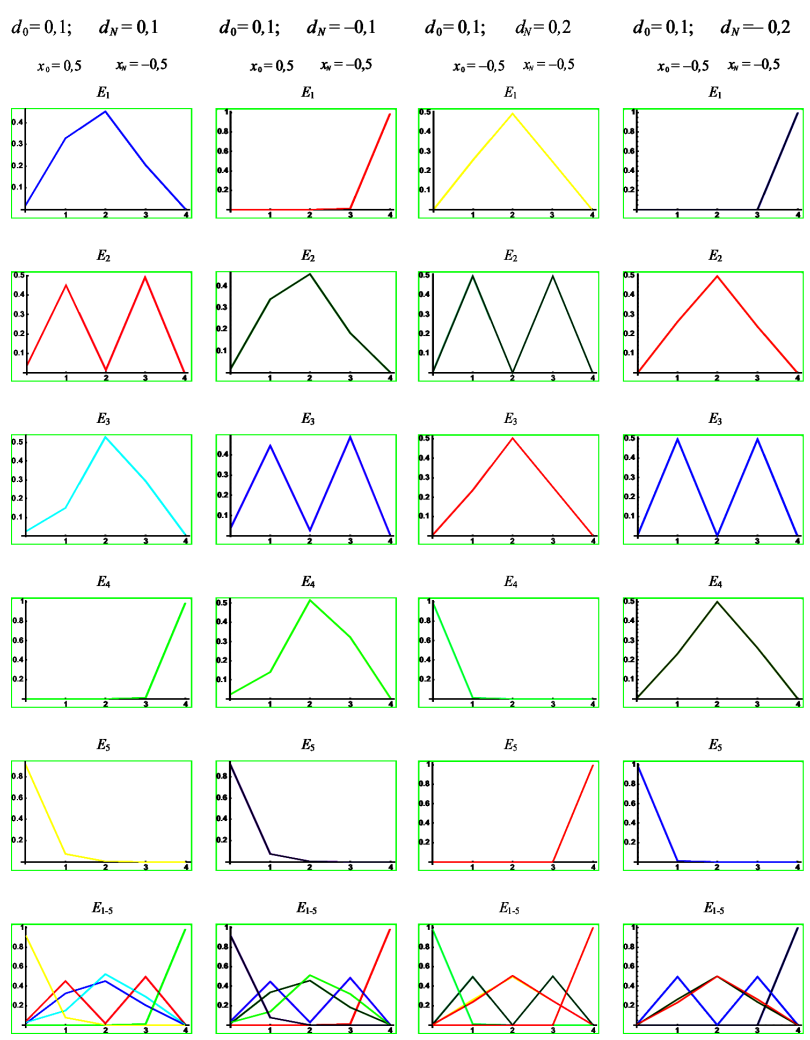

Fig. 6. Space distribution of exciton states in ultrathin molecular film.
TABLE I

Probabilities of finding excitons in four-layered film.

\begin{tabular}{|c|c|c|c|c|c|}
\hline \multirow{2}{*}{$\begin{array}{c}\text { Reduced } \\
\text { relative } \\
\text { energy }\end{array}$} & \multicolumn{5}{|c|}{$d_{0}=d_{N}=0.0 ; x_{0}=0.0 ; x_{N}=0.0$} \\
\hline & 1st plane & 2nd plane & 3rd plane & 4th plane & 5 th plane \\
\hline-1.73205 & & & & & \\
\hline 0 & & & & & \\
\hline 0.00000 & & & & & 333 \\
\hline & & & & & \\
\hline & & & & & \\
\hline \multirow{2}{*}{$\begin{array}{c}\text { Reduced } \\
\text { relative } \\
\text { energy }\end{array}$} & \multicolumn{5}{|c|}{$d_{0}=0.2 ; d_{N}=0.0 ; x_{0}=0.0 ; x_{N}=0.0$} \\
\hline & 1st plane & ane & 3rd plane & lane & 5 th \\
\hline-1.63052 & & & & & \\
\hline & & & & & \\
\hline & & & & & 77 \\
\hline & & & & & \\
\hline 10. & & & & & $0^{-9}$ \\
\hline \multirow{2}{*}{$\begin{array}{c}\text { Reduced } \\
\text { relative } \\
\text { energy }\end{array}$} & \multicolumn{5}{|c|}{$d_{0}=d_{N}=0.2 ; x_{0}=x_{N}=0.0$} \\
\hline & 1 st $\mathrm{F}$ & ane & 3rd & lane & 5th plane \\
\hline-1.45855 & & & & & 0195 \\
\hline-0.09901 & J485 & & & & 0.00485 \\
\hline & & & 0.51712 & & 0.00318 \\
\hline & & & & & 0.4 \\
\hline 10.10099 & 0.49485 & 0.00504 & 0.000019 & 0.00504 & 0.49485 \\
\hline
\end{tabular}

\begin{tabular}{|c|c|c|c|c|c|}
\hline \multirow{2}{*}{$\begin{array}{c}\text { Reduced } \\
\text { relative } \\
\text { energy }\end{array}$} & \multicolumn{5}{|c|}{$d_{0}=d_{N}=0.0 ; x_{0}=2.0 ; x_{N}=0.0$} \\
\hline & $1 \mathrm{st}$ & d pla & 3rd plar & 4th plane & 5th plat \\
\hline-3.18168 & & & & & $8 T$ \\
\hline-1.3 & 579 & Ju & 21079 & 54 & 0.26242 \\
\hline 0.0 & & & & & \\
\hline 1.37 & & 00 & & & 442 \\
\hline 3.18 & 39 & 0.49254 & 37 & & 0.0 \\
\hline $\begin{array}{l}\text { Reduced } \\
\text { relative }\end{array}$ & \multicolumn{5}{|c|}{$d_{0}=d_{N}=0.0 ; x_{0}=x_{N}=2.0$} \\
\hline energy & ne & & & & \\
\hline-3.31663 & 54 & & & 00 & 54 \\
\hline 3.0 & 0.2 & & & & 000 \\
\hline 0.00 & & & 0.8 & & 090 \\
\hline 3.00 & 00 & & & 00 & 0.25000 \\
\hline 3.3 & & & & & 454 \\
\hline $\begin{array}{l}\text { Reduced } \\
\text { relative }\end{array}$ & \multicolumn{5}{|c|}{$0=d_{N}=0.3 ; x_{0}=x_{N}=2.0$} \\
\hline energy & 1st plane & 2nd & 3rd & lane & 5th plane \\
\hline-1.70891 & & & & & 0.00938 \\
\hline-0.5 & & & & & 788 \\
\hline 1.12653 & 91 & & 0.6 & & 0.00891 \\
\hline 15.5 & 0.48211 & & 000 & .01788 & 0.48211 \\
\hline 15.58238 & 0.48169 & 0.01815 & 0.00029 & 0.01815 & 0.48169 \\
\hline
\end{tabular}

Green functions (5.7), we obtain

$$
\varepsilon_{n_{z}}^{-1}=1-\frac{S}{2} \sum_{\nu=1}^{N+1} \sum_{s=+,-} \frac{g_{n_{z}}^{\nu}}{\varrho_{s}-\varrho_{\nu}},
$$

where $\varrho_{ \pm}=\mp|f|-|p|-\mathcal{F}_{x y}$, and by arranging this expression it finally follows: 


$$
\varepsilon_{n_{z}}(\omega)=\left[1+S \sum_{\nu=1}^{N+1} g_{n_{z}}^{\nu} \frac{\varrho_{\nu}-|p|+\mathcal{F}_{x y}}{|f|^{2}-\left(\varrho_{\nu}-|p|+\mathcal{F}_{x y}\right)^{2}}\right]^{-1} .
$$

Based on this and formula (5.8) it is clear that relative permittivity depends on position, e.g. number of crystallographic planes $\left(n_{z}=0,1,2, \ldots, N\right)$ along the direction of broken translational symmetry by existing of border areas (perpendicular onto them), of course and on nondimensional energy/frequency of external electromagnetic field. These functional dependences are mathematically very complicated and can be determined only numerically. Beside that, in this case we have 5 (e.g. 6) parameters: $N=4\left(n_{z}=0,1,2,3,4\right), d_{0 / N} \in\{-0.3,+0.3\}$ and $x_{0 / N} \in\{-0.8,+2.0\}$, which means that we have 6000 different combinations of their possible values. That is performed with the help of adequate software (see Appendix for corresponding code lines) and is graphically arranged.
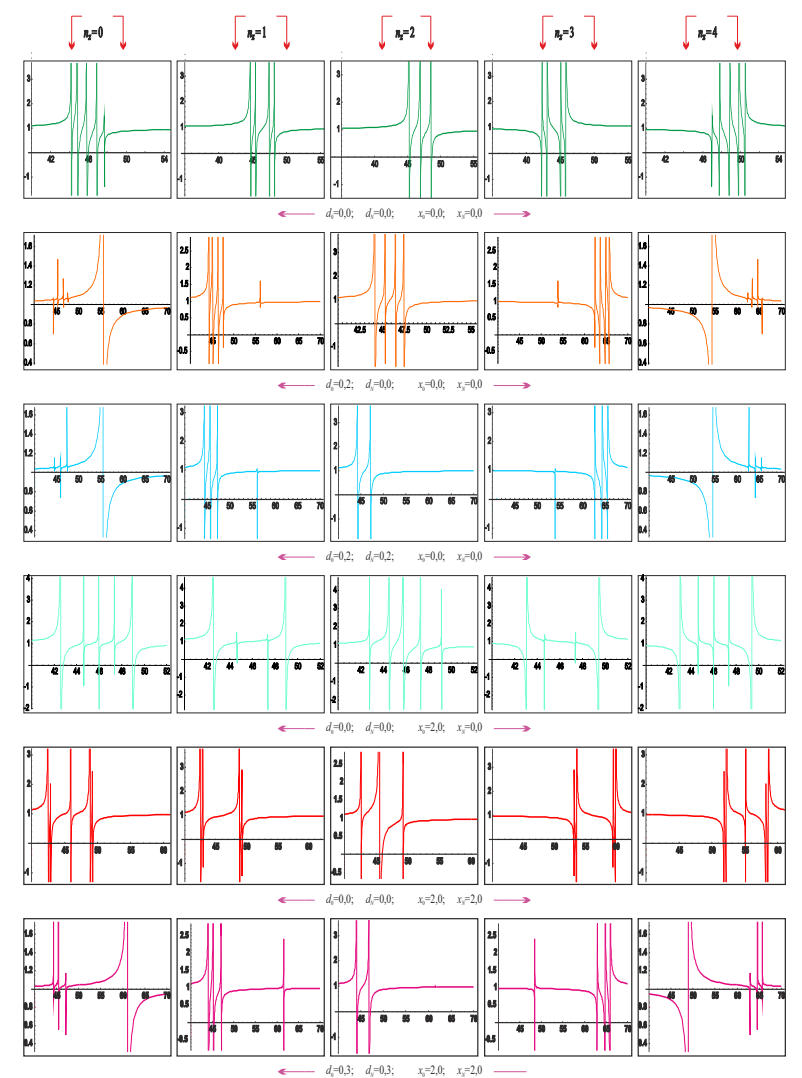

Fig. 7. Dynamic permittivity of molecular film.

Figure 7 shows the dependence of dynamic permittivity $(\varepsilon)$ on reduced relative energy of external electromagnetic field $(f \equiv \hbar \omega /|X|)$, for four-layered molecular film. Dependence was calculated for a center of plane (2D) Brillouin's zone $\left(k_{x}=k_{y}=0\right)$, but individually per plane (parallel border areas) of crystalline film, therefore for $n_{z}=0$ and/or 4 , then $n_{z}=1$ and/or 3 and finally for $n_{z}=2$.

For each graph there is shown the influence of border parameters $d_{0, N}$ and $x_{0, N}$ both on the permittivity size and shape of its dependence on external field frequency, and on the number and position of resonant peaks $\mathbb{I}$. It is clearly visible that there is a final number of resonant peaks, since $k_{z}$ component of exciton wave vector is discrete. Only for certain values of $k_{z}$ (for given values of $k_{x}$ and $k_{y}$ ) resonant phenomena and radiation absorption may occur.

In the observed film $(N=4)$ the number of resonant peaks is different for different layers. Therefore, at least three and a maximum of five are present as much as there are allowed states along axis where translation symmetry is violated, in this case along $z$-axis). Their number depends on the size of perturbation parameters at border areas, i.e. in the border layers of a crystalline film and this is in direct connection to the occurrence of localized states for some determined values of border parameters.

First upper figure with 3 graphs shows the permittivity of the ideal ultra thin film with 4 inter-atomic layers. In the second and third rows, Fig. 7 shows the dependence of dynamic permittivity on reduced frequency of outer electromagnetic field for four-layered dielectric film, when parameter $\Delta$ is changed by perturbation $d_{0}$ or $d_{N}$.

From this it is visible that a number of resonant peaks, i.e. resonant lines (when $\varepsilon\left(\omega_{r}\right) \rightarrow \pm \infty$ ) depends on the number, i.e. position of atomic plane $n_{z}$ for which permittivity is being calculated, but also from value of parameter $d_{0 / N}$. By increasing parameter $d_{0 / N}$ widening of absorption zone occurs, and dominating resonant peak is visible only in the border area of the film $\left(n_{z}=0\right.$ or $\left.n_{z} \equiv N=4\right)$. This is expected, since analysis of spectral weights for exciton states shows that probability of finding an exciton in the localized state is higher at exactly the same spot.

In the fourth and fifth row the dependence of dynamic permittivity $\varepsilon$ on reduced energy $f$ of four-layered dielectric film is shown, when parameter is $\left.x_{0} / N\right)$. It is visible that a number of resonant peaks for permittivity is different for every layer, i.e. $\varepsilon=\varepsilon\left(n_{z}\right)$, but it also depends on parameter $x_{0 / N}$. After increasing value of this parameter, the symmetric widening of absorption zone occurs towards both higher and lower values of energy for all layers, but also some resonant peaks are being quenched depending on position (film layer) for which permittivity is determined. For instance, all resonant peaks exist only for the border $\left(n_{z}=0\right.$ and 4$)$ and middle plane $\left(n_{z}=2\right)$, while in other planes they are quenched.

In the last row of figures dependence of this film permittivity on frequency of outer field is shown, when all border parameters are the most perturbed. It is evident that the influence of perturbation parameter $x$ is silenced

I Resonant peaks in the frequency dependence of dynamic permittivity are the positions resonant frequencies, where permittivity diverges into $\pm \infty$. Those are also energies (wavelengths) of such electromagnetic radiance that a model crystal "swallows" at the given place, i.e. energies that are absolutely absorbed there. Frequency of the outer field at which resonant peaks occur is obtained from condition $\varepsilon_{n_{z}} \rightarrow \infty$. 
and that dominating behavior is dictated by perturbation $d$. Here two resonant absorptions occur in pair both on border areas, but their distance is bigger than without parameter $x$.

The subject of this research is not new and obtained results are in agreement with results applied in [12]. In contrast, in paper [12] there was introduced a new approach for calculation of the optical absorption spectra of organic polycrystalline thin films.

In particular, a new relation for molecular excitation energy shifts due to dispersion effects of the environment has been derived. The obtained formulae allow us to determine level shift of the molecule from the site, depending of the given nanoscale environment.

On that basis there could be explained dissimilarity of the double structure transition in the absorption spectrum of PDCTI films by the coexistence of two molecular phases. Large crystals, where most molecules are located in the interior, give rise to the component on the low-energy side. However, the blue shift is generally non-structured function, due to a phase of loosely packed small aggregates which pass trough a weaker gasto-crystal phase, as most molecules reside at or close to the surface.

Therefore, these results explain the absorption properties as disorder induced by the dispersive effects due to non-resonant exciton coupling. In previous works $[12,21]$ these properties are explained by strong resonant exciton coupling in such a way that oscillator transfers power on vibration modes. While a fully quantitative analysis of the film morphology is beyond the scope of this work, our findings emphasize that an explicit consideration of the local energy structure is mandatory to understand the optical properties of molecules in nano scaled solid-state systems.

The polarized absorption spectra of crystalline pentacene are obtained in [22] for excitation normal to the $a b$ herringbone plane by measuring transmitted light in ultrathin crystals. The spectral line shapes for excitation polarized along $b$ and orthogonal to $b$ are analyzed theoretically using a Holstein-like Hamiltonian which includes both the Frenkel and charge transfer (CT) excitons represented in a multi-particle basis set. The model agrees with prior estimates regarding the strong CT contribution $(\approx 45 \%)$ of the exciton responsible for the $b$-polarized lower Davydov component. The polarization resolution allows one to also establish the nature of the upper Davydov component, which is found to contain far less CT content $(\approx 15 \%)$, as well as the nature of the higherenergy vibronic excitons, which are found to consist of a complex mixture of the Frenkel one- and two-particle states and CT excitons. Generally, the spectrum polarized along $b$ displays $J$-aggregate-like vibronic signatures while the spectrum polarized orthogonal to $b$ displays $H$ aggregate-like vibronic signatures. The result is entirely consistent with the calculated exciton band dispersion which is in good agreement with experimental measurement.
The absorption spectrum corresponding to the solution and crystalline phase of pentacene is shown in [22]. Dramatic spectral changes appear after the crystallization process. The two orthogonally polarized components are shown, which has been obtained by rotating the polarizer through $90^{\circ}$. The polarizer angle which maximizes the peak at $14700 \mathrm{~cm}-1$ coincides with the electric field polarization along the crystallographic $b$-axis, according to the ellipsometry and reflectivity calculations [23].

New nanomaterial - squaraines - has been introduced [24] which offers significant potential for organic photovoltaic devices due to their broad absorbance and high extinction coefficients as well as their expected usage as mechanic probes in such devices. In this work are taken all necessary steps to develop a comprehensive understanding of the excited-state properties of squaraines based on their molecular structure and the resulting solid-state packing. Accurate locations of the absorption spectral peaks are calculated on the basis of an essential model of states, which has been expanded to include intermolecular charge transfer (ICT). A comparison of simulated spectra with spectra for two symmetric squaraine derivatives confirms that ICT has a major influence on the optical and electronic properties of squaraine aggregates, fully accounting for the origin of the strong panchromaticity of these systems.

Comparing our research results with those obtained in $[22-25]$, which correspond to the near UV, visible and deep IC region of electromagnetic field, one can conclude good agreement for resonant absorption, which could be attributed and explained with spatial and boundary conditions, i.e. with effect of quantum confinement and nanoscale dimensions of structures, exciton coupling and overall dynamic of excitons. Different number and shape of absorption lines appear due to the fact that we have included only linear exciton spectra, while vibrational and rotational contributions are neglected. Furthermore, an important conclusion is that here are well defined positions of the central relative permittivity peaks and optical indices from the external electromagnetic field. Relative differences in compared spectra come from the different crystal samples (chemical or physical) which dictate difference in collective absorption process and redistribution of exciton energy.

\section{Conclusion}

This paper describes the theoretical investigation of specificity in microscopic and macroscopic properties of exciton systems in the molecular ultra thin film structures. Most important results of these analysis are as follows:

1. The energy spectrum of possible exciton energy was determined:

(a) The dispersion exciton spectrum in the films is discrete, and number of discrete levels is equal to number of atomic planes of film along the 
axis of spatial limitation. In the bulk there exists only one zone, where excitons "take on" all possible energy values.

(b) The energy change of molecules at the nodes of border planes causes movement spectrum towards higher energy levels, and this movement is happening in such a way that one or two energy levels are "expelled" out of the bulk zone, giving localized states.

(c) Increasing transfer energy of excitons in the border layers causes movement of exciton energy spectrum outside bulk borders, "expelling" two and even four energy levels. These localized states are more distanced from the bulk zone as perturbation is higher.

2. Contrary to the bulk structures, where excitons can be found at any position with equal probability, in the molecular film structures probabilities to find excitons strongly depend on the film thickness and intensity of parameter change at the border areas of the film:

(a) Whether exciton will be separated at the border areas of a film depends on the perturbation energy of molecules in those layers. The increase of this energy proportionally increases probability of exciton localization at the border areas, and these excitons have higher possible energies.

(b) Localization of excitons between border and adjoining planes is determined by changes of transfer energy of excitons between borders of these layers. The increase of these parameters causes the increase of probability to find excitons in the border areas of the film considered.

3. In the molecular film structures, resonant peaks exist at precisely determined energies. Number of these peaks depends on position of planes in the film for which permittivity is calculated, but also from the size of perturbation parameters:

(a) By changing energy values for molecules at the border areas, absorption zone widens, and dominating resonant peaks are visible only at those border areas.

(b) By increasing vault energy of excitons in the border layer of the film, symmetrical widening of absorption zone occurs, and also quenching certain inner resonant peaks.

\section{Acknowledgments}

This work was partially supported by the Ministry of Education, Science and Technological Development of the Republic of Serbia (Grant No. OI-171039) and by the Provincial Secretariat for Science and Technological Development of Vojvodina (114-451-927) as well as by the Ministry of Science and Technology of the Republic of Srpska (19/6-020/961-16/15).

\section{Appendix A: Calculation of roots}

In an IDEAL ULTRATHIN FILM there are no perturbations of border parameters (i.e. $d_{0}=d_{N}=0$, $x_{0}=x_{N}=0$; "cut-off" case), so that the determinant (4.1) represents Chebychev's polynomial [12-14]:

$$
\mathcal{D}_{N+1}(\varrho)=\mathcal{C}_{N+1}(\zeta)=\frac{\sin (N+2) \zeta}{\sin \zeta}, \quad \varrho=2 \cos \zeta,
$$

where Chebychev's polynomials satisfy the known recurrent relationship of the form: $\mathcal{C}_{n+1}(x)=x \mathcal{C}_{n}(x)-$ $\mathcal{C}_{n-1}(x)$. From the condition (4.1), i.e. for $\mathcal{C}_{N+1} \equiv 0$, we obtain: $\zeta_{\nu}=\frac{\pi \nu}{N+2}$, where $\nu=1,2,3, \ldots, N+1$. On the basis of this and Eq. (3.4), we find

$$
\hbar \omega_{\nu}=\Delta-2|X|\left(\cos a k_{x}+\cos a k_{y}-\cos \frac{\pi \nu}{N+2}\right) .
$$

In comparison with the dispersion law of excitons in the bulk we will write this expression in simplified and dimensionless form $\left(\varrho_{\nu}=-2 \cos a_{z} k_{z}(\mu)\right)$ :

$$
\begin{aligned}
\mathcal{E}_{k_{x} k_{y}}(\mu / \nu) & =\mathcal{F}_{x y}+\mathcal{G}_{z}(\mu / \nu), \\
\mathcal{G}_{z}(\mu / \nu) & =\frac{1}{2}\left[1-\cos a_{z} k_{z}(\mu)\right] \equiv \frac{2+\varrho_{\nu}}{4} .
\end{aligned}
$$

Previous expression is the law of excitons dispersion of the ideal molecular film and has the same form as the expression for the energy of excitons corresponding ideal not limited structure [7-9], with the difference that there $k_{z}$ is virtually continuous variable (in the interval $[0, \pi / a])$ as well as $k_{x}$ and $k_{y}$, and here is discrete and is given by the expression

$$
k_{z}(\mu)=\frac{\pi}{a} \frac{\mu}{N+2} .
$$

where next change of indices is introduced $\mu=N+1-\nu$, $\mu=1,2,3 \ldots, N+1$.

\section{Appendix B: Necessary software package}

Calculation and graphic presentation of the:

1. possible exciton energy states

2. probability and spatial distribution of exciton states

3. frequency dependence of dynamical permittivity

1. $\mathrm{d}=$.

$$
\begin{aligned}
& \mathrm{d}[\mathrm{n}]]:=\mathrm{r}^{*} \mathrm{~d}[\mathrm{n}-1]-\mathrm{d}[\mathrm{n}-2] \\
& \mathrm{d}[0]:=1 \\
& \mathrm{~d}[-1]:=0 \\
& \mathrm{~d}[-2]:=0 \\
& \mathrm{~d}[-3]:=0 \\
& \mathrm{~d}[-4]:=0 \\
& \mathrm{~d}[1]:=\mathrm{r} \\
& \mathrm{d}[2]:=\mathrm{r}^{2}-1 \\
& \mathrm{n}=4
\end{aligned}
$$


Frac $=50(*$ "Frac" represents the ratio of energy at node and energy transfer $*$ )

"At this point Chebychev polynomials are defined and the number of film layers is set $(\mathrm{n}) "$

$\mathrm{e} 0=0(*$ Set the value of -0.3 to $+0.3 *)$

"Repair of exciton energy at the bottom border area"

en $=0(*$ Set the value of -0.3 to $+0.3 *)$

"Repair of exciton energy at the upper border area"

$\mathrm{v} 0=0(*$ Set the value of -0.8 to $+2.0 *)$

"Repair of exciton energy transfer at the bottom border areas"

$\mathrm{vn}=0(*$ Set the value of -0.8 do $+2.0 *)$

"Repair of exciton energy transfer at the upper border area" $\mathrm{k}=$.

$\mathrm{r}=$.

$\mathrm{m}=$.

$\mathrm{s}=$.

$\operatorname{Do}\left[\left\{\mathrm{k}=\frac{j \pi}{10}, \mathrm{rr}=\mathrm{N}\left[\operatorname{Solve}\left[\left(\mathrm{r}-\right.\right.\right.\right.\right.$ Frac $\left.^{*} \mathrm{e} 0\right)\left(\mathrm{r}-\right.$ Frac $\left.^{*} \mathrm{en}\right) \mathrm{d}[\mathrm{n}-1]$

- $\left(\left(\mathrm{r}-\right.\right.$ Frac $\left.^{*} \mathrm{e} 0\right)(1+\mathrm{vn})^{2}+\left(\mathrm{r}-\right.$ Frac*en $\left.^{*}(1+\mathrm{v} 0)^{2}\right) \mathrm{d}[\mathrm{n}-2]$

$\left.\left.+(1+\mathrm{v} 0)^{2}(1+\mathrm{vn})^{2} \mathrm{~d}[\mathrm{n}-3]==0, \mathrm{r}\right]\right], \mathrm{rrr}=$

Table[Re[Sort[r/.rr]]],

$\mathrm{s}[\{\mathrm{j}\}]=$ Table $[\{\mathrm{N}[-4 \operatorname{Cos}[\mathrm{k}]], \mathrm{N}[\operatorname{rrr}[[\mathrm{i}]]-4 \operatorname{Cos}[\mathrm{k}]]\},\{\mathrm{i}, 1, \mathrm{n}+$ $1\}], \operatorname{Print}[\mathrm{s}[\{\mathrm{j}\}]]$,

$\mathrm{m}=$ Table $[\{\mathrm{s}[\{0\}], \mathrm{s}[\{1\}], \mathrm{s}[\{2\}], \mathrm{s}[\{3\}], \mathrm{s}[\{4\}], \mathrm{s}[\{5\}], \mathrm{s}[\{6\}]$,

$\mathrm{s}[\{7\}]$,

$\mathrm{s}[\{8\}], \mathrm{s}[\{9\}], \mathrm{s}[\{10\}]\}]\},\{\mathrm{j}, 0,10\}]$

(* $\mathrm{j}$ is the number of points by which the dispersion lines are drawn. 10 are selected. Equation is solved in which the Chebyshev polynomials are described, and solutions are the energies r. That energies are then sorted and form the matrices $\mathrm{s}$ and $\mathrm{m}$ in which pairs of points define the dispersion lines in non dimensional form. ${ }^{*}$ )

Print $[\mathrm{m}]$

$\mathrm{g}=$ Transpose $[\mathrm{m}]$

Show[gg1 $=$ ListPlot[g[[1]], PlotJoined $\rightarrow$ True], gg2 = ListPlot[g[[2]], PlotJoined $\rightarrow$ True],

gg3 = ListPlot[g[[3]], PlotJoined $\rightarrow$ True $],$ gg4 $=$

ListPlot[g[[4]], PlotJoined $\rightarrow$ True],

gg5 = ListPlot[g[[5]], PlotJoined $\rightarrow$ True $], \mathrm{q}=\operatorname{Plot}[\{-2+\mathrm{x}$, $2+\mathrm{x}\},\{\mathrm{x},-4,4\}$,

PlotStyle $\rightarrow$ Dashing $[\{.03, .03\}]\}]$, PlotRange $\rightarrow$ Automatic]

2. $\mathrm{b} 0[0]=1$

$\mathrm{b} 0\left[\mathrm{~m}_{-}\right]:=(\mathrm{r}-$ Frac e 0$) \mathrm{d}[\mathrm{m}-1]-(1+\mathrm{v} 0)^{2} \mathrm{~d}[\mathrm{~m}-2]$

$\mathrm{bn}[0]=1$

$\mathrm{bn}\left[\mathrm{m} \_\right]:=(\mathrm{r}-$ Frac en $) \mathrm{d}[\mathrm{m}-1]-(1+\mathrm{vn})^{2} \mathrm{~d}[\mathrm{~m}-2]$

$\mathrm{Dd}=(\mathrm{r}-$ Frac e0 $)(\mathrm{r}-$ Frac en $) \mathrm{d}[\mathrm{n}-1]-((\mathrm{r}-$ Frac e0 $)$

$(1+\mathrm{vn})^{2}+(\mathrm{r}-$ Frac en $\left.)(1+\mathrm{v} 0)^{2}\right) \mathrm{d}[\mathrm{n}-2]+(1+\mathrm{v} 0)^{2}(1$

$+\mathrm{vn})^{2} \mathrm{~d}[\mathrm{n}-3]$

$\mathrm{dd}=\partial_{\mathrm{r}} \mathrm{Dd}$

$\mathrm{rr}=\mathrm{N}[$ Solve $[\mathrm{Dd}==0, \mathrm{r}]]$

rrr $=$ Table[Re[Sort[r /. rr]]]

(* Calculation of tabular data *)

$\operatorname{Do}\left[\left\{\mathrm{r}=\operatorname{rrr}[[\mathrm{j}]], \quad \mathrm{s}[\{\mathrm{j}\}]=\operatorname{Table}\left[\left\{\mathrm{m}, \quad \mathrm{N}\left[\frac{1}{100000}\right.\right.\right.\right.\right.$

Round $\left.\left.\left[\frac{100000 \mathrm{~b} 0[\mathrm{~m}] \mathrm{bn}[\mathrm{n}-\mathrm{m}]}{\mathrm{dd}}\right]\right]\right\}$,

$\{\mathrm{m}, 0, \mathrm{n}\}], \operatorname{Print}[\mathrm{s}[\{\mathrm{j}\}]]\},\{\mathrm{j}, 1, \mathrm{n}+1\}]$

$11=$ ListPlot $[\mathrm{s}[\{1\}]$, PlotJoined $\rightarrow$ True $]$

$12=$ ListPlot $[\mathrm{s}[\{2\}]$, PlotJoined $\rightarrow$ True $]$

$13=$ ListPlot $[\mathrm{s}[\{3\}]$, PlotJoined $\rightarrow$ True $]$

$14=$ ListPlot $[\mathrm{s}[\{4\}]$, PlotJoined $\rightarrow$ True $]$
$15=$ ListPlot $[\mathrm{s}[\{5\}]$, PlotJoined $\rightarrow$ True $]$

(*Drawing grids, and summary data*)

Show $[11,12,13,14,15$, PlotRange $\rightarrow$ Automatic]

$===$

(* $\mathrm{m}$ represents $\mathrm{nz}$-th plan for which we calculate permittivity,

we will see that, for example, for five-layered film results are matching with

$\mathrm{nz}=0$ and 4 or for $\mathrm{nz}=1 \mathrm{i} 3$, and for center plane $\mathrm{nz}=2$ is different $*$ )

$\operatorname{Do}\left[\left\{\mathrm{r}=\operatorname{rrr}[[\mathrm{j}]], \mathrm{s}[\{\mathrm{j}\}]=\right.\right.$ Table[N $\left.\left[\mathrm{b} 0[\mathrm{~m}]^{*} \mathrm{bn}[\mathrm{n}-\mathrm{m}] / \mathrm{dd}\right]\right]$,

$\operatorname{Print}[\mathrm{s}[\{\mathrm{j}\}]]\},\{\mathrm{j}, 1, \mathrm{n}+1\}]$

$\mathrm{ss}[\mathrm{n}]:=\mathrm{s}[\mathrm{n}]^{*}\left(\right.$ Frac $\left.-4+\operatorname{rrr}[[\mathrm{n}]]+\mathrm{I}^{*} \operatorname{Sir}\right) /\left(\mathrm{w}^{2}-(\right.$ Frac $-4+$ $\left.\left.\operatorname{rrr}[[\overline{\mathrm{n}}]]+\mathrm{I}^{*} \mathrm{Sir}\right)^{2}\right)$

$\mathrm{ee}=(1+\mathrm{ss}[1]+\mathrm{ss}[2]+\mathrm{ss}[3]+\mathrm{ss}[4]+\mathrm{ss}[5])^{-1}$

$\operatorname{sse}\left[\mathrm{n}_{-}\right]:=\mathrm{s}[\mathrm{n}]^{*}($ Frac $-4+\operatorname{rrr}[[\mathrm{n}]]) /\left(\mathrm{w}^{2}-(\right.$ Frac $-4+$ $\left.\operatorname{rrr}[[\mathrm{n}]])^{2}\right)$

ees $=(1+\operatorname{sse}[1]+\operatorname{sse}[2]+\operatorname{sse}[3]+\operatorname{sse}[4]+\operatorname{sse}[5])^{-1}$

Print["PERMITTIVITY"]

(* drawing of single and total data *)

$\mathrm{k} 0=$ Plot $[$ ees, $\{\mathrm{w}, 30,65\}$, PlotRange $\rightarrow\{\{30,65\}$,

Automatic $\}]$

(* permittivity $*$ )

\section{Appendix C: Spatial distribution of probability}

In the case of non-perturbed film, subsidiary determinants $B_{n_{z}-1}$ and $B_{N-n_{z}}$ are reduced to Chebyshev's polynomials $\mathcal{C}_{n_{z}} \wedge \mathcal{C}_{N-n_{z}}$. Then the Green functions and spectral weights have the following form:

$$
G_{n_{z}}=\frac{\mathrm{i} \hbar}{2 \pi|X|} \frac{\mathcal{C}_{n_{z}} \mathcal{C}_{N-n_{z}}}{\mathcal{C}_{N+1}}, \quad g_{n_{z}}^{\nu}=\frac{\mathcal{C}_{n_{z}}\left(\varrho_{\nu}\right) \mathcal{C}_{N-n_{z}}\left(\varrho_{\nu}\right)}{\left.\frac{\mathrm{d}}{\mathrm{d} \varrho} \mathcal{C}_{N+1}(\varrho)\right|_{\varrho=\varrho(\nu)}} .
$$

In an ideal film there exist only volume states of excitons. Finding probability can be found analytically. Using rule for derivative of the determinant we obtain

$$
\begin{aligned}
& {\left[\frac{\mathrm{d}}{\mathrm{d} \varrho} \mathcal{C}_{N+1}(\varrho)\right]_{\varrho=\varrho_{\nu}}=\sum_{i=1}^{N+1} \mathcal{C}_{i-1}\left(\varrho_{\nu}\right) \mathcal{C}_{N+1-i}\left(\varrho_{\nu}\right)=} \\
& \sum_{i=1}^{N+1} \frac{\sin i \xi_{\nu} \sin (N+2-i) \xi_{\nu}}{\sin ^{2} \xi_{\nu}}
\end{aligned}
$$

and spectral weights become

$$
\begin{gathered}
g_{n_{z}}^{\nu}=\frac{\sin \left(n_{z}+1\right) \xi_{\nu} \sin \left(N-n_{z}+1\right) \xi_{\nu}}{\sum_{i=1}^{N+1} \sin i \xi_{\nu} \sin (N+2-i) \xi_{\nu}} \equiv \\
\frac{\sin ^{2}\left(n_{z}+1\right) \xi_{\nu}}{\sum_{i=1}^{N+1} \sin ^{2} i \xi_{\nu}}
\end{gathered}
$$

(by introducing replacement $\xi_{\nu}=\frac{\pi \nu}{N+2}$ ). The sum of the denominator can be solved by using known series expand: $\sum_{k=1}^{m} \sin ^{2} k x=\frac{m}{2}-\frac{\cos (m+1) x \sin m x}{2 \sin x}$, it is obtained: $\sum_{i=1}^{N+1} \sin ^{2} i \xi_{\nu}=\frac{N+2}{2}$. Probabilities of finding excitons are then:

$$
g_{n_{z}}^{\nu}=\frac{2}{N+2} \sin ^{2}\left(\left(n_{z}+1\right) \frac{\pi \nu}{N+2}\right) .
$$




\section{References}

[1] L.L. Chang, L. Esaki, Phys. Today 45, 36 (1992).

[2] M.C. Tringides, M. Jalochowski, E. Bauer, Phys. Today 60, 50 (2007).

[3] K. Wood, P. Hammond, D. Schmidt, S. Wrightman, B. Andaya, Thin Film Delivers Drugs, Biophotonics, www.photonics.com; W. Frasch, D. Spetzler, Tiny Biosensor to Detect DNA, Biophotonics, www.photonics.com.

[4] M.G. Cottam, D.R. Tilley, Introduction to Surface and Superlattice Excitations, University Press, Cambridge 1989 .

[5] S.G. Davison, M. Steslicka, Basic Theory of Surface States, Oxford Press, Clarendon 1996.

[6] M. Prutton, Introduction to Surface Physics, Oxford Press, Clarendon 1984; Clarendon 1994.

[7] V.M. Agranovich, Theory of Excitons, Nauka, Moskva 1968 (in Russian).

[8] V.M. Agranovich, V.L. Ginzburg, Crystal Optic with Space Dispersion and Theory of Excitons, Nauka, Moskva 1979 (in Russian).

[9] A.A. Maradudin, in: Surface Polaritons, Eds. V.M. Agranovich, D.L. Mills, North-Holland, Amsterdam 1982, p. 405; Light Scattering and Nanoscale Surface Roughness, Ed. A.A. Maradudin, in: Nanostructure Science and Technology, Ed. D.J. Lockwood, Springer, New York 2007.

[10] S. Lazarev, Ž.M. Škrbić, J.P. Šetrajčić, D.Lj. Mirjanić, Lj. Ristovski, J. Phys. Chem. Solids 58, 793 (1997).

[11] V.D. Sajfert, J.P. Šetrajčić, D. Popov, B.S. Tošić, Physica A 353, 217 (2005).

[12] I.D. Vragovich, J.P. Šetrajčić, R. Scholz, Eur. Phys. J. B 66, 185 (2008).
[13] S.S. Pelemiš, J.P. Šetrajčić, B. Markoski, N.V. Delić, S.M. Vučenović, J. Comput. Theor. Nanosci. 6/7, 1474 (2009).

[14] J.P. Šetrajčić, arXiv:1004.2387, [cond-mat-sci], 2010.

[15] V.M. Agranovich, B.S. Toshich, Zh. Eksp. Teor. Fiz. 53, 149 (1967) [ Sov. Phys.-JETP 26, 104 (1968)].

[16] R.P. Djajić, D.Lj. Mirjanić, B. Nikin, J.P. Šetrajčić, B.S. Tošić, J. Phys. C 20, 5585 (1987).

[17] E.N. Economou, Green's Functions in Quantum Physics, Springer, Berlin 1949.

[18] G. Rickayzen, Green's Functions and Condensed Matter, Academic Press, London 1980.

[19] G. Mahan, Many Particle Physics, Plenum Press, New York 1990.

[20] I.E. Dzyaloshinskii, L.P. Pitaevskii, Zh. Eksp. Teor. Fiz. 36, 1797 (1959) [Sov. Phys. JETP 9, 1282 (1959)].

[21] I.D. Vragovich, R. Scholz, J.P. Šetrajčić, Mater. Sci. Forum 518, 41 (2006).

[22] N.J. Hestand, H. Yamagata, B. Xu, D. Sun, Yu Zhong, A.R. Harutyunyan, G. Chen, H.-L. Dai, Yi Rao, F.C. Spano, J. Phys. Chem. C 119, 22137 (2015).

[23] D.C. Qi, H.B. Su, M. Bastjan, O.D. Jurchescu, T.M. Palstra, A.T.S. Wee, M. Rubhausen, A. Rusydi, Appl. Phys. Lett. 103, 113303 (2013).

[24] N.J. Hestand, C. Zheng, A.R. Penmetcha, B. Cona, J.A. Cody, F.C. Spano, C.J. Collison, J. Phys. Chem. C 119, 18964 (2015).

[25] J. Megow, T. Körzdörfer, T. Renger, M. Sparenberg, S. Blumstengel, F. Henneberger, V. May, J. Phys. Chem. C 119, 5747 (2015). 